\title{
Objectivity, culturelessness, and apoliticism: how cultural beliefs prevent the advancement of equity in astronomy graduate programs
}

\author{
Fatima N. Abdurrahman \\ Department of Astronomy, University of California, Berkeley, CA, USA 94720 \\ Alice R. Olsmtead \\ Department of Physics, Texas State University, San Marcos, TX, USA 78666
}

\begin{abstract}
After decades of interest in promoting diversity and inclusion in the field and higher education more broadly, physics and astronomy departments in American universities remain predominantly white and male. While some STEM fields have made progress in recent years towards correcting the historic overrepresentation of white men, $75 \%$ of physics and astronomy $\mathrm{PhDs}$ awarded in 2019 went to this demographic that constitutes only $30 \%$ of the U.S. population, higher than chemistry, math, computer science, and engineering [1]. This work seeks to understand the barriers to creating a more diverse field by examining how its cultural beliefs can work to maintain inequity in graduate programs.

In this paper, we present a subset of the results from a larger study which used the lived experiences of 12 female and gender-nonconforming students of color to understand how physics and astronomy graduate programs in American predominantly white institutions maintain equity gaps between majoritized and minoritized students. This paper focuses on the theme of equity work in departments, and how it relates to a foundational cultural belief in Western physics and astronomy: that physicists comprise an objective, cultureless, and apolitical community, impervious to social influence [2]. By contextualizing participants' observations of attitudes, policies, and behaviors with the literature, we attempt to demonstrate a how departments can systematically prevent the advancement of equity goals, and subsequently, create negative outcomes for minoritized students. We emphasize the design of program structures as a critical point of intervention, and conclude the work with recommendations based on participant input.
\end{abstract}

2021 PERC Proceedings edited by Bennett, Frank, and Vieyra; Peer-reviewed, doi.org/10.1119/perc.2021.pr.Abdurrahman Published by the American Association of Physics Teachers under a Creative Commons Attribution 4.0 license. Further distribution must maintain the cover page and attribution to the article's authors. 


\section{INTRODUCTION}

Despite accounting for only $30 \%$ of the US population, $75 \%$ of U.S. PhDs in physics and astronomy in 2019 were awarded to white men. In contrast, women of underrepresented racial minorities (URM[3]) earned less than $3 \%$ of the same PhDs while comprising $15 \%$ of the U.S. population [1]. The field exists in this grossly unrepresentative state decades into growing interest in supporting women and underrepresented minorities, with research, educational initiatives, and entire careers being dedicated to this goal [4]. While adjacent fields such as chemistry and biology have made significant strides towards achieving equity goals in this time, physics maintains one of the lowest percentages of bachelors, masters, and doctoral degrees awarded to underrepresented minorities in the STEM fields, second only to astronomy. Similarly, both fields remain far from achieving gender parity at any level of higher education [5].

While much discussion of equity goals focuses on the presumed deficits of minoritized students or metrics of representation, growing evidence indicates the manifestation of systems of oppression (e.g., white supremacy, patriarchy, and others) in institutions of higher education as being a barrier, as well as the institutional actors that maintain them [6]. Notions of the reduced abilities of minoritized students have been demonstrated to be negatively influential in the admissions, teaching, and advising of students [7-9]. Further, female URM graduate students in astronomy are 15-20 times more likely to report discrimination or harassment than nonURM males [10]. Both gender and race influence the persistence of graduate students in astronomy, suggesting that the internal operations of graduate programs (rather than gatekeeping alone) reduce the presence of minoritized scientists in the field [10].

This paper draws on results from a larger study which sought to understand how cultural beliefs in the academic astronomy and physics community maintain inequity in graduate programs. In this work, we focus on the theme of equity work in departments, and how it relates to a foundational cultural belief in Western physics and astronomy: that physicists comprise an objective, cultureless, and apolitical community, impervious to social influence [2].

\section{BACKGROUND: CULTURAL BELIEFS}

This work focuses on cultural beliefs, or related patterns of attitudes and behaviors maintained by a group and passed down to new group members [11]. We can think of the cultural beliefs of the physics and astronomy community as the stories told in this community about how physics is done, who physicists are, and what makes a great physicist. As most of the literature focuses on the culture of physics rather than astronomy, we assume that the latter has inherited similar cultural beliefs as the former. Additionally, the culture of academia in these specific fields in the U.S. is inextricable from the broader culture of contemporary Western universities, particularly predominantly white institutions (PWIs). Thus, we are considering communities that sit at the intersection of physics and academia in a Western context.

We focus on one belief in this work: that physicists are immune to the influences of culture due to their objectivity [2]. This idea is explained by Harding: "[The physicists"] nonsocial subject matter and the paradigmatic status of their methods appear to preclude critical reflection on social influences on their conceptual systems; indeed, prevalent dogma holds that it is the virtue of modern science to make such reflection unnecessary" [12]. However, this belief does harm to Black physicists who understand that, rather than disappearing in physics spaces, racism is amplified by collective denial stemming from the belief that physics is cultureless [13]. This mirrors 'colorblind' racial ideology in contemporary American society, which purports to support diversity while failing to address the existence of racism [14]. Thus, putative 'culturelessness' obfuscates the genuine presence of systems of oppression inherited from broader American culture.

This image of 'culturelessness' is held up by the physicist's dedication to the ideal of objectivity. In this ideal, impartiality and a focus on evidence take precedence over personal preference or feeling. The perseverance of 'objectivity' prioritizes men in the field. Historical studies of science indicate that modern universities were intentionally established in association with the 'masculine' qualities of intellectual rigor and objectivity, while distancing itself from the 'feminine' qualities of emotion and subjectivity [15]. The deeply social nature of what is considered to be 'objective' is described as "claims that are held up as 'objective' [that] are actually 'socially negotiated'.. through the interaction of particular individuals with particular cultural, social, and cognitive values" [16].

Relatedly, there is an emerging veneer of 'apoliticism' in physics communities. 'Politics' broadly refers to "who gets what, when, and how," which includes the sociopolitical aspects of equity work in education [17]. An aversion to the 'political' is present across higher education. For example, white admissions counselors are more likely to respond to deracialized, apolitical black students than black students who demonstrate a commitment to racial justice [18]. An aversion to politics is also evident in how diversity work is approached in many physics departments. Some physicists feel that they should not be involved in political discussion, while others recognize the necessity of addressing broader social issues in the work of addressing diversity in STEM [19]. For example, Cochran finds that some faculty members are comfortable with \#BlackLivesMatter, while others are not. This makes addressing anti-blackness a matter of personal opinion and debate [19]. We draw a parallel between 'culturelessness' and 'apoliticism' as they both seek to present their holders as neutral parties, while preventing the underlying biases of the field (which are influenced by culture and inherently political) from being challenged. 


\section{METHODS}

Participants for this work were recruited via emails sent to chairs of 200 physics and astronomy departments and professional societies (e.g., SACNAS), as well as on social media via hashtags (e.g., \#BlackInAstro). The criteria were 1) having both minoritized gender and racial identities (URM or otherwise); and 2) that they have finished at least three years of a PhD program at an American PWI. The sample contained 8 URM participants, 3 gender-nonconforming participants, 6 participants with non-heterosexual orientations, 5 participants from low-income backgrounds, 5 participants who practice a non-dominant (i.e., non-Christian) religion, and 2 international students, for a total of 12 participants. The participants ranged in age from 26-34, with two having completed their PhDs within a year of interview. All participants were involved in astronomy research (whether in an astronomy department or an astronomy group within a physics department) at R1 or R2 universities, in programs with normative times to completion of 5-7 years. Pseudonyms will be used for all participants.

The data presented in this work were collected in individual, semi-structured, 1-2 hour long interviews conducted and recorded over Zoom in February 2021. Questions were openended, focusing on topics including department culture, othering and belonging, and academic experiences. The interview included an exercise in which participants rated 22 personality traits on a scale from 'most negative' (-3) to 'most positive' (+3) with the prompt 'Traits you believe your department values in its graduate students.' For findings in Section IV to which a specific trait corresponded, the mean and uncertainty (standard deviation over square root of sample size) of the rating from -3 to +3 are reported alongside interview data. Interview data were iteratively analyzed using in vivo coding to best capture the perspective of the participants. At the highest level, codes were organized into attitudes, program structures, faculty practices, and student outcomes.

\section{FINDINGS}

\section{Attitudes: Valuing Apoliticism}

Seven out of twelve participants discussed their departments' value of being 'apolitical', with three reporting that they were told to "not be political." Sarah describes such an interaction with her department's graduate advisor:

She has told a number of students when [we asked]... 'What sorts of things do you have on campus that will support students of color, or women of color?' And one of her comments is, 'Oh, don't get political... Just focus on your research. Don't think about that at all.'

Topics participants said were labeled 'political' were largely issues relating to minoritized identities, such as anti- blackness in departments follwing the \#BlackLivesMatter protests of Summer 2020. Nur elaborates on these specific definition of 'apolitical', saying:

Usually it depends who I'm talking to what 'apolitical' even means. If I'm talking to a white, wealthy, male faculty, 'political' can mean something as trivial as talking about trans rights or talking about having a gender neutral restroom. That, to them, can seem very political.

Consistently, participants rated 'apolitical' as a positively valued trait by their departments $(1.25 \pm 0.36)$.

\section{Program Structure: Ineffective Codes of Conduct}

A structure that demonstrates the influence of this attitude in our data set was department codes of conduct. Five participants reported largely ineffectual codes of conduct, with one participant saying their department does not even have a code of conduct. Mariam described a faculty member who made racist and transphobic remarks in classes and colloquia despite "a robust code of conduct," with no action taken aside from "limiting their intersection with students":

[We don't have] clear pipelines for repercussions, for breaking this supposed code of conduct that's supposed to help us... There's absolutely nothing on there about what happens if you do those things... There were several grad students, especially more junior ones, who would come in, see the code of conduct, feel protected by it, then watch someone break it, and try to do something about it. And nothing happens.

\section{Faculty Practice: Avoiding DEI Issues}

Seven participants described faculty members who avoid discussing problems that exist in their departments and encourage students to do the same. Ferdous said:

It's very difficult to point out the flaws that are in the department when the people who are in leadership... don't want to rock the boat. Don't want to acknowledge that there might be issues. Don't want to acknowledge that the way they've been doing things can be improved. Especially when that department [is highly ranked], they think that they're doing something right.

This avoidance is consistent with the ranking of the trait 'confrontational', which participants ranked as the most negatively valued trait by their departments $(-2.33 \pm 0.67)$. 


\section{Faculty Practice: Discouraging DEI Work}

Seven participants discussed faculty members actively discouraging DEI work. Ferdous, like two others, was told to prioritize research:

They don't want us to have any other stake in anything else [besides research]... [Faculty ask,] 'Why are you spending all this time doing nonresearch things? Going to conferences, trying to recruit or talk to other minority students about your experience? And why are you doing these things that are not science related?'

In some cases, observed attitudes towards DEI were not simply deprioritization, but outright antagonism, with Marwa observing a faculty member on an admissions committee describing applicants interested in DEI work as "entitled." In other cases, participants described feeling "tone policed" or "gaslit" when discussing racism or other systems of oppression. This discouragment of DEI work is consistent with participants' ranking of the trait 'activist', which they ranked as highly negative in their department's values $(-2.0 \pm 0.58)$.

\section{Faculty Practice: Performatively Promoting DEI}

Though all participants mentioned some DEI efforts existing in their departments, nine found some of these efforts performative. This was observed in many forms, the most frequent being the performance of symbolic efforts that lacked follow through. For example, several participants described their departments publishing a statement in reaction to \#BlackLivesMatter affirming a commitment to combating racism in a department, but failing to fulfill those commitments. Mariam describes her case, with an extra dimension of plagiarism:

\section{A bunch of grad students and postdocs wrote [a statement]. And then [the faculty] took it, took out the things that were too extreme for them, and then published it as their own...And not only that, they obviously haven't done anything that they said they would do on that piece of paper that they watered down to begin with.}

Participants who discussed these "broken promises" of performative DEI efforts report it happening both as departmentwide projects (e.g., statements) and in one-on-one interactions with faculty. Four participants encountered faculty members who simultaneously presented themselves as 'allies' in public (e.g., on Twitter) while treating minoritized students poorly in private. Other examples of performative DEI work included 'bait-and-switch' recruitment, tokenizing minoritized students while not supporting them adequately, focusing DEI efforts as outreach rather than internally, only engaging in DEI work in response to department crises, and
DEI efforts uninformed by an intersectional lens (e.g., gender equity catering to white women).

\section{Student Outcomes: "Suffering in Silence"}

A common outcome of the practices described above was an educational environment in which students with minoritized identities were effectively unprotected from discrimination and harassment, whether from faculty, peers, or other department members. This is echoed by all but one participant reporting at least one instance (though usually multiple instances) of microaggressions related to race, gender, or other minoritized identities from department members. These microaggressions included racist, sexist, or otherwise discriminatory remarks from faculty and exclusion from academic and social activities by fellow grads. There was additionally a general sense that, because of the lack of channels for reporting harassment and overall lack of interest in rectifying such problems, there was no recourse aside from simply dealing with it. Mabrooka describes the pressure to accept inequitable treatment, which she observed acting both on herself and some peers:

If you never point out a flaw in someone or ask for something to be different, it might help you to succeed...Any sort of feedback that calls into question [faculty's] perfect advising or their perfect teaching or whatever, it's viewed as a negative on the person who's [giving feedback]... [Students] just suffer in silence, terrified to say anything.

This circumstance of "suffering in silence" was common, with five students similarly describing struggling with interpersonal problems and feeling unable to seek solutions.

\section{DISCUSSION}

The enlightenment ideal of the disembodied, transcendent scientist observing nature without bias or emotion has become an essential characteristic of the archetypal physicist, positioning it as the purest form of knowledge acquisition and sharing its aura of rationality with those who are perceived as similar to the scientists of yore [20]. It follows that in a country where the field remains dominated by the same demographic that produced such ideals (namely, white men), modern physicists extend their faith in the objectivity of their science to their understanding of people who do science [12].

In Section IV 1, we found students were encouraged to "not be political." By naming discussions of subjects like affinity-based support groups or bathroom access for gendernoncomforming folks as 'political,' faculty members create boundaries defining what is and is not relevant to doing science, positioning the concerns of marginalized groups as irrelevant. It follows that, if concerns of socially-influenced 
misconduct are irrelevant to doing science, creating robust structures for accountability and conflict resolution are similarly unimportant. The lack of effective codes of conduct in nearly half of our sample's departments (Section IV 2) is consistent with this, and creates an environment where students see faculty members as unaccountable actors.

Most participants in this work found it difficult to buy into what they perceive as false images of inclusive departments, and often reported taking it upon themselves to try improve department climate. However, these efforts were often blocked; in Section IV 4, we described how participants were discouraged from engaging in DEI oriented work by faculty. While faculty were observed to treat DEI work as an extra, possibly unnecessary part of the job, participants who endured misconduct due to their identities saw it as a foundational piece of being able to do the job at all. This becomes especially worrying considering the ubiquity of misconduct discussed in Section I.

While all participants' departments communicated in some form they value DEI, all participants had experiences that were inconsistent with this stated value. This relates back to the studies described earlier, showing similar tensions to Cohran and Boveda's physics professors who are uncomfortable saying Black Lives Matter and Thornhill's white admissions counselors who favor 'apolitical' Black students over 'political' ones. Several participant accounts were strikingly similar to how Carbado and Gulati describe employers desiring aesthetic 'diversity' without attending to the complexities of a diverse population: "'Good blacks' will think of themselves as people first and black people second (or third or fourth); they will neither 'play the race card' nor generate racial antagonism or tensions in the workplace; they will not let white people feel guilty about being white; and they will work hard to assimilate themselves" [21]. This practice of selecting assimilated 'diversity' (or expecting the existing 'diversity' to assimilate themselves) allows departments to satisfy the social and legal requirements of including minoritized people, without having to do the difficult work of creating an equitable environment, all the while accruing what Carbado and Gulati called the "diversity benefit."

Participants observed a similar phenomenon in faculty who perform the motions of DEI work without meaningfully changing the treatment of minoritized groups, as described in Section IV 5. Sara Ahmed explains how documents stating commitments to anti-racism or similar values are often used as symbolic gestures of inclusion that get counted as having done the work of creating inclusive work environments [22]. Her description of diversity work as being "about changing perceptions of whiteness rather than changing the whiteness of organizations" parallels most participants' observation of DEI work in their departments: performatively valuing and seeking diverse students, without meaningfully changing the cultural norms of the department. This performative dynamic can also be seen in the behaviors of individual faculty members; as 'diversity statements' or 'broader impacts' are a required component of many job applications and grant pro- posals in the field, there is strong incentive for faculty members to cultivate an image of supporting and contributing to DEI work. However, as our participants explain in Section IV 5, some faculty members publicly present an inclusive image (for example, via social media) while treating minoritized students poorly in private. Regardless of intention, this way of enacting DEI work has the potential to be damaging; as Ahmed said, "self-perception of being good at communication and care blocks the recognition of racism" [22].

Participants who spoke of performative DEI work generally characterized that work as lacking an intersectional frame, that is, being unable to identify and treat the unique circumstances of multiply minoritized groups. The necessity of this type of intersectional analysis was initially discussed by Dr. Kimberlé Crenshaw, who identified how being simultaneously black and female in certain workplaces allowed for a type of discrimination that did not affect black men or non-black women [23]. A study in astronomy and planetary science observed Crenshaw's "double-jeopardy" to exist within these fields, finding that women of color are significantly more likely than white women, white men, or men of color to experience harassment or assault in the workplace on the basis of their gender or race [24]. In our study, many participants felt alienated by groups and people meaning to support them along one axis of identity while being apathetic or hostile towards other components of their identity.

\section{CONCLUDING RECOMMENDATIONS}

To counter the barriers discussed above, we have compiled a list of recommendations to consider in efforts to attain equitable graduate programs. These recommendations are based on participant input as well as existing literature. They are:

- Increase transparency and communication with students in all possible aspects of their programs [25]

- Orient hiring and admissions towards building a "critical mass' of diverse students and faculty [5]

- Provide equity-oriented faculty training, especially in mentoring, leadership, and interpersonal skills [25]

- Create, communicate, and enforce robust codes of conduct and channels for reporting misconduct [26]

- Build community within departments between students, faculty, and staff [27]

- Decentralize advising from the model of one advisor, one student, to flexible, team, or holistic advising [28]

- Design mentorship structures between peers and across professional levels

- Recognize and reward equity work alongside research or teaching achievements [29]

- Enact service requirements for graduate students and faculty to distribute the DEI workload

- Utilize research-based methods for equitable pedagogy and department management (e.g., PER literature). 
[1] N. C. for Science and N. S. F. Engineering Statistics, Doctorate recipients from u.s. universities: 2019 (2019).

[2] S. Traweek, Beamtimes and lifetimes (Harvard University Press, 1988).

[3] Note that URM is defined here as African American/Black, Hispanic/Latinx, Native American/Alaskan Native, Native Hawaiian/Other Pacific Islander, Filipino, Hmong, Vietnamese, or two or more races, with at least one from the preceding list.

[4] R. Beichner, An introduction to physics education research, in Getting Started in PER, Vol. 2 (2009).

[5] J. R. Posselt, Equity in Science: Representation, Culture, and the Dynamics of Change in Graduate Education (Stanford University Press, 2020).

[6] E. M. Bensimon, Closing the achievement gap in higher education: An organizational learning perspective, New directions for higher education 2005, 99 (2005).

[7] C. W. Miller, B. M. Zwickl, J. R. Posselt, R. T. Silvestrini, and T. Hodapp, Typical physics ph. d. admissions criteria limit access to underrepresented groups but fail to predict doctoral completion, Science Advances 5, eaat7550 (2019).

[8] E. A. Canning, K. Muenks, D. J. Green, and M. C. Murphy, Stem faculty who believe ability is fixed have larger racial achievement gaps and inspire less student motivation in their classes, Science advances 5, eaau4734 (2019).

[9] K. G. Wilkins-Yel, J. Bekki, A. Arnold, B. Bernstein, C. Okwu, M. Natarajan, and A. K. Randall, Understanding the impact of personal challenges and advisor support on stem persistence among graduate women of color., Journal of Diversity in Higher Education (2021).

[10] R. Ivie, Results from the longitudinal study of astronomy graduate students, in American Astronomical Society Meeting Abstracts\# 223, Vol. 223 (2014) pp. 304-03.

[11] E. Hatah, K. P. Lim, A. M. Ali, N. M. Shah, and F. Islahudin, The influence of cultural and religious orientations on social support and its potential impact on medication adherence, $\mathrm{Pa}-$ tient preference and adherence 9, 589 (2015).

[12] S. G. Harding, The science question in feminism (Cornell University Press, 1986).

[13] S. Hyater-Adams, C. Fracchiolla, T. Williams, N. Finkelstein, and K. Hinko, Deconstructing black physics identity: Linking individual and social constructs using the critical physics identity framework, Physical Review Physics Education Research 15, 020115 (2019).

[14] I. X. Kendi, How to be an antiracist (One world, 2019).
[15] M. Ong, Body projects of young women of color in physics: Intersections of gender, race, and science, Social problems $\mathbf{5 2}$, 593 (2005).

[16] A. Harell, S. Soroka, and S. Iyengar, Race, prejudice and attitudes toward redistribution: A comparative experimental approach, European Journal of Political Research 55, 723 (2016).

[17] H. D. Lasswell, Politics: Who gets what, when, how (Pickle Partners Publishing, 2018).

[18] T. Thornhill, We want black students, just not you: How white admissions counselors screen black prospective students, Sociology of Race and Ethnicity 5, 456 (2019).

[19] G. L. Cochran and M. Boveda, A framework for improving diversity work in physics, in Physics Education Research Conference 2020, Virtual Conference. Retrieved April, Vol. 19 (2020) p. 2021.

[20] D. Haraway, Situated knowledges: The science question in feminism and the privilege of partial perspective, Feminist studies 14, 575 (1988).

[21] D. W. Carbado and M. Gulati, Acting white?: Rethinking race in post-racial America (Oxford University Press, 2013).

[22] S. Ahmed, Ethnic and Racial Studies 30.4, 590 (2007).

[23] K. Crenshaw, Demarginalizing the intersection of race and sex: A black feminist critique of antidiscrimination doctrine, feminist theory and antiracist politics, u. Chi. Legal f. , 139 (1989).

[24] K. Clancy, Journal of Geophysical Research: Planets 122.7, 1610 (2017).

[25] M. Aly, The key to a happy lab life is in the manual, Nature 561, 7 (2018).

[26] Z. Rezaee, R. C. Elmore, and J. Z. Szendi, Ethical behavior in higher educational institutions: The role of the code of conduct, Journal of business ethics 30, 171 (2001).

[27] J. Posselt, K. Reyes, K. Slay, A. Kamimura, and K. Porter, Equity efforts as boundary work: How symbolic and social boundaries shape access and inclusion in graduate education, Teachers College Record 119, 1 (2017).

[28] B. A. Polnarieve, R. Jaafar, T. Hendrix, H. P. Morgan, P. Khethavath, and A. B. Idrissi, Nourishing stem student success via a team-based advisement model., International Journal of Higher Education 6, 31 (2017).

[29] H. B. Carlone and A. Johnson, Understanding the science experiences of successful women of color: Science identity as an analytic lens, Journal of Research in Science Teaching: The Official Journal of the National Association for Research in Science Teaching 44, 1187 (2007). 